Radial and Nonradial Pulsations as Probes of Stellar Physics

ASP Conference Series, Vol. 259, 2002

C. Aerts, T.R. Bedding, \& J. Christensen-Dalsgaard, eds.

\title{
A Spectroscopic Search for Non-Radial Pulsations in Early B-Type Stars
}

\section{Schrijvers}

Astronomical Institute Anton Pannekoek, University of Amsterdam, and Center for High Energy Astrophysics, Amsterdam, Netherlands

\author{
J.H. Telting
}

Nordic Optical Telescope, Apartado 474, E-38700 Santa Cruz de La Palma,Spain; jht@not.iac.es

\section{J. De Ridder}

Instituut voor Sterrenkunde, Katholieke Univ. Leuven, Celestijnenlaan 200B,B-3001Leuven, Belgium; joris@ster.kuleuven.ac.be

It is known that early-B type main-sequence stars can be unstable against pulsations induced by the $\kappa$ mechanism. Traditionally, radial and low-degree non-radial $\beta$ Cephei-like pulsations have been detected photometrically; nowadays radial, low-degree and intermediate-degree pulsations can be detected spectroscopically through line-profile variations (LPVs). Whereas most of the brightest early-B type stars have been photometrically monitored for variability (72 of the 541 O9.5-B3 III-V stars in the BSC are classified as $\beta$ Cephei stars), a systematic LPV survey to look for pulsational behaviour was lacking. Here we present some preliminary results of the southern part of our spectroscopic survey of early-B type stars. We have made a high spectral resolution survey of the Si III $\lambda 4552,4567,4574 \AA$ triplet in early-B type stars in order to look for LPVs due to non-radial pulsation (NRP). Our sample consists of 82 southern O9.5-B3 stars with spectral classes III-V, taken from the BSC; $27 \%$ of all 09.5-B2.5 III-V stars in the BSC were sampled. This sample is not fully representative for all nearby early-B stars, because we concentrated on stars with high rotational velocities, because many of the stars are members of the Sco-Cen associations, and because we focused on the $\beta$ Cephei stars in the BSC. As much as $40 \%$ of the $\beta$ Cephei stars in the BSC with $v \sin i \geq 25 \mathrm{~km} \mathrm{~s}^{-1}$ were sampled. The observations were carried out with the ESO CAT telescope at resolution $R \sim 65000$. Our targets were observed during 6 runs in September 1995 - June 1998. Some stars were observed many times, but most targets were observed only once or twice.

We have concentrated on detecting LPVs in the form of Doppler-mapped quasi absorption and emission bumps that are typical for NRP in stars with rotationally broadened lines. We used the presence of such bumps in the Si lines as an indicator of the likely presence of NRP. For stars with only one spectrum we checked for the clear presence of bumps in the available lines of the Si triplet. For stars with more than one spectrum we checked whether the LPVs caused by the moving bumps resemble that of NRP. Line shifts and broadenings were taken as indicators for low-degree pulsations $(\ell \leqslant 2)$. For stars in which we detected LPV, follow-up observations are needed to confirm the presence of NRP. 
Table 1. Targets with detected LPV. Values of $v \sin i$ indicated with ${ }^{*}$ were estimated from our spectra, others are from the BSC. Last column: ' $\mathrm{r}$ ' rectification problems; ' $\mathrm{b}$ ' possible mis-identification due to binarity; '2' double-lined binary; ' $\mathrm{n}$ ' $\mathrm{S} / \mathrm{N}$ too low to be conclusive.

\begin{tabular}{|c|c|c|c|c|c|c|c|c|c|c|c|}
\hline object & $v \sin i$ & $\mathrm{~N}$ & bumps & degree & & object & $v \sin i$ & & bumps & degree & \\
\hline$\alpha \operatorname{Lup}$ & 24 & 2 & & lo & $\mathrm{b}$ & $\beta$ Cen & 139 & 4 & yes & hi & 2 \\
\hline$\nu$ Eri & 25 & 3 & & 10 & & $\psi^{2}$ Ori & 141 & 1 & yes & hi & 2 \\
\hline$\kappa$ Cen & 28 & 7 & yes & hi & $b$ & $\omega^{1}$ Sco & 142 & 89 & yes & hi & \\
\hline V357 Car & 30 & 9 & & lo & $\mathrm{b}$ & V372 Car & 147 & 25 & yes & hi & \\
\hline QU Pup & $\sim 30^{*}$ & 2 & & lo & & $\alpha$ Mus & 147 & 14 & yes & hi & \\
\hline$\theta \mathrm{Oph}$ & 35 & 4 & & lo & & 7 Mon & 152 & & suspect & hi & $\mathrm{b}, \mathrm{n}$ \\
\hline$\alpha$ Tel & 35 & 19 & yes & hi & & $\rho$ Sco & 156 & 2 & yes & hi & \\
\hline$\alpha \mathrm{Pav}$ & 39 & 2 & & lo & $b, n$ & $\alpha$ Vir & 159 & 3 & yes & hi & \\
\hline$\mu^{1}$ Cru & 48 & 1 & suspect & hi & $\mathrm{n}$ & $\epsilon$ Cen & 159 & 539 & yes & hi & \\
\hline EY CMa & 49 & 2 & yes & hi/lo & & HR 3089 & 160 & & suspect & hi & $\mathrm{n}$ \\
\hline QS Pup & $\sim 50^{*}$ & 2 & suspect & hi & $\mathrm{n}$ & HR 3359 & 163 & & suspect & hi & \\
\hline$\sigma$ Sco & 53 & 5 & & lo & & $\lambda$ Sco & 163 & 26 & yes & hi & \\
\hline$\mu^{2}$ Sco & 57 & 1 & yes & hi & $b$ & VV Ori & 168 & 1 & suspect & hi & b \\
\hline$\zeta \mathrm{CMa}$ & 63 & 3 & suspect & hi/lo & $\mathrm{b}, \mathrm{n}$ & HR 3294 & 169 & 46 & yes & hi & \\
\hline HR 7029 & 65 & 4 & yes & hi/lo & b & HR 6960 & 172 & 3 & yes & hi & \\
\hline HR 3358 & 66 & 36 & yes & hi & & HR 3293 & 178 & 13 & yes & hi & \\
\hline$v$ Sco & 73 & 10 & yes & hi & 2 & HR 3453 & 181 & & suspect & hi & $\mathrm{n}, \mathrm{b}, 2$ \\
\hline HR 6143 & 90 & 6 & yes & hi & & $\delta$ Sco & 181 & 121 & yes & hi & \\
\hline$\nu$ Cen & 91 & 98 & yes & hi & & QZ Pup & 187 & & suspect & hi & $\mathrm{n}$ \\
\hline$\gamma \mathrm{Col}$ & 96 & 1 & suspect & hi & & $\delta$ Cru & 194 & 18 & yes & hi & \\
\hline$\pi \mathrm{Sco}$ & 100 & 1 & suspect & hi & $\mathrm{b}, 2$ & HR 3819 & 207 & 19 & yes & hi & 2 \\
\hline V2052 Oph & 120 & 7 & & lo & & OS Pup & 213 & 2 & yes & hi & \\
\hline$v^{1}$ Cen & 122 & 3 & suspect & hi & $n$ & $\delta$ Lup & 221 & 2 & yes & hi & \\
\hline$\phi$ Cen & 126 & 6 & yes & hi & & $\sigma$ Cen & 245 & & suspect & hi & $r$ \\
\hline$\beta$ Lup & 127 & 85 & yes & hi & & HX Vel & 285 & & suspect & hi & $\mathrm{r}, \mathrm{b}, \mathrm{n}$ \\
\hline$\beta^{1} \mathrm{Sco}$ & 130 & 2 & suspect & hi & $\mathbf{n}$ & HR 3476 & 288 & & suspect & hi & $\mathrm{r}, \mathrm{b}, \mathrm{n}$ \\
\hline$\kappa$ Sco & 131 & 18 & yes & hi & & 23 Ori & 295 & & suspect & hi & $\mathrm{r}, \mathrm{b}, \mathrm{n}$ \\
\hline$\epsilon$ Lup & 133 & 3 & yes & hi & 2 & & & & & & \\
\hline
\end{tabular}

The Doppler-mapped NRP bumps are most apparent for pulsating stars with rotation velocities $50<v \sin i<250 \mathrm{~km} \mathrm{~s}^{-1}$. For lower rotation velocities the broadening is not high enough to be able to resolve the stellar disc in velocity space; for higher rotation velocities the lines of the Si III triplet are so shallow and broad that the detection of bumps in just one or two spectra is very difficult because of $\mathrm{S} / \mathrm{N}$ limitations and errors in continuum normalization. In Be stars with strong disc emission the region of the SiIII triplet is polluted by Fe II emission lines, hampering easy detection of NRP in the Si lines.

We find that 40 out of the 57 stars with $50<v \sin i<250 \mathrm{~km} \mathrm{~s}^{-1}$ in our sample show evidence for LPVs. For stars that were not previously known to have a $\beta$ Cep/NRP nature, and that have $50<v \sin i<250 \mathrm{~km} \mathrm{~s}^{-1}, 26$ out of 37 show NRP-like bumps. This most probably indicates that many early-B type stars are pulsationally excited. 